\title{
Una acción decidida contra la sepsis
}

\section{A decisive action against sepsis}

\author{
Fabian Alberto Jaimes-Barragán • Medellín (Colombia)
}

\author{
"Caminante, son tus huellas \\ el camino y nada más; \\ Caminante, no hay camino, \\ se hace camino al andar"
}

(ANTONIO Machado. Extracto de proverbios y cantares)

Hace una década encontramos que cuatro de cada 100 admisiones por urgencias en hospitales de Colombia se debían a infecciones bacterianas que amenazaban la vida, y uno de cada cinco pacientes hospitalizados podía estar en esa misma condición (1). En esa misma población de estudio, si se presentaba disfunción de algún órgano o shock, la mortalidad a los 28 días podía alcanzar 22 y $46 \%$, respectivamente. Nada nos hace suponer que hoy, salvo algunas diferencias marginales en las cifras, el panorama sea sustancialmente diferente. Las infecciones bacterianas agudas y graves, lo que formalmente llamamos sepsis, siguen siendo una causa excesivamente frecuente de enfermedad, hospitalización, muerte y discapacidad en Colombia y en el mundo (2-5).

¿Por qué razón ocurre esto y qué podemos hacer al respecto? Las respuestas pueden ser muchas e incluso contradictorias; pero una perogrullada parece estar en la base de todo: no podemos resolver un problema si ni siquiera lo reconocemos como tal. Apenas en 2017, la Asamblea Mundial de la Salud y la Organización Mundial de la Salud (WHA/WHO) hicieron de esto una prioridad de salud global, al adoptar una resolución para mejorar la prevención, el diagnóstico y la atención clínica de la sepsis (http://www.who.int/sepsis/en/). Un cambio de paradigma fundamental, si pasamos de simplemente aproximarnos como médicos al cuidado agudo de estos pacientes, a ver un problema de salud pública que requiere soluciones sistémicas y basadas en la población. La complejidad de la sepsis impide siquiera pensar en una única estrategia que se ajuste a todos los escenarios. Las diferencias dependerán de los microorganismos locales, los factores de riesgo preponderantes en la población, las intervenciones existentes y la disponibilidad de recursos, entre muchos otros. Esto quiere decir que en cada país donde ciudadanos mueran por esta causa, los gobiernos, los sistemas y trabajadores de la salud, las universidades y la comunidad en general, deben emprender acciones y adoptar actitudes que reflejen la búsqueda de soluciones ajustadas a las particularidades de cada región, pero eficaces y efectivas en una meta final común: no más muertes, ni secuelas evitables, por culpa de la sepsis.

La declaración de São Paulo, que se publica en este número de la revista y se difunde actualmente en otros medios de América Latina (http://www.ilas.org.

Dr. Fabián Alberto Jaimes-Barragán: MSc, $\mathrm{PhD}$, Profesor Titular, Departamento de Medicina Interna, Universidad de Antioquia. Director de Investigaciones, Hospital San Vicente Fundación. Medellín (Colombia). Correspondencia. Dr. Fabian Jaimes B. Medellín (Colombia).

E-mail: fabian.jaimes@udea.edu.co Recibido: 30/VII/2018 Aceptado: 13/IX/2018 
br/see-declaration.php. Sepsis: la principal causa de muerte y discapacidad prevenibles en América Latina. Un llamado a la acción para reducir el impacto de la sepsis), es un paso en esa dirección. En Colombia, desde la Universidad de Antioquia y la Fundación Hospitalaria San Vicente de Paúl, con sus sedes de Medellín y Rionegro; hemos convocado al Ministerio de Salud y Protección Social, a la Asociación Colombiana de Facultades de Medicina (ASCOFAME), a la Asociación Colombiana de Medicina Crítica y Cuidado Intensivo (AMCI), a la Asociación Colombiana de Medicina Interna (ACMI), a la Asociación Colombiana de Infectología (ACIN) y a la Asociación Colombiana de Especialistas en Urgencias y Emergencias (ACEM); para que hagamos juntos, como el inmenso Antonio Machado, camino al andar.

\section{Referencias bibliográficas}

1. Rodriguez F, Barrera L, De La Rosa G, Dennis R, Duenas C, Granados M, et al. The epidemiology of sepsis in Colombia: a prospective multicenter cohort study in ten university hospitals. Critical care medicine. 2011;39(7):1675-82. Epub 2011/06/21.

2. Fleischmann C, Scherag A,Adhikari NK, Hartog CS, Tsaganos T, Schlattmann P, et al. Assessment of Global Incidence and Mortality of Hospital-treated Sepsis. Current Estimates and Limitations. American journal of respiratory and critical care medicine. 2016;193(3):259-72. Epub 2015/09/29.

3. Finfer S, Machado FR. The Global Epidemiology of Sepsis. Does It Matter That We Know So Little? American journal of respiratory and critical care medicine. 2016;193(3):228-30. Epub 2016/02/02.

4. Jaimes F, Ascuntar J, Hincapie C, Valencia M, Vallejo C, Moncayo G, et al New clinical criteria for septic shock are valid and reproducible: An independent prospective cohort. Critical care medicine. 2016;44(12):417.

5. Jaimes F, Leon A, Ascuntar J, Niño C, Londoño J, Plaza M, et al. Prospective validation of qSOFA in emergency services: A useless bedside clinical score. Critical care medicine. 2016;44(12):429. 